\title{
La participation au service de la régulation urbaine dans le cadre des projets urbains et patrimoniaux à Gênes, Italie et à Valparaiso, Chili
}

Sébastien Jacquot, Paris

\section{La participation, catégorie polysémique de l'action publique et de l'analyse}

Dans un contexte post-fordiste et post-keynésien émergent de nouveaux paradigmes de l'aménagement, dans lesquels les territoires locaux et les villes prennent une part accrue (LE GALEs 2003). Parallèlement se développe une «injonction participative» (SinTOMER \& BLONDIAUX 2002) ou un «paradigme participatif» (BACQUE, REY \& SinTOMER 2005), aboutissant à la mise en place d'outils d'implication des citoyens dans les politiques urbaines, encouragée dans les pays du Sud par les organisations internationales (LEGRos 2008).

La participation peut être définie comme une modalité de l'action collective (BLATRIX 2002) consistant en l'inscription d'acteurs locaux non publics dans des programmes d'aménagement, qu'ils agissent comme habitants, citoyens ou porte-parole de groupements. Elle désigne à la fois une injonction politique et une catégorie d'analyse des politiques publiques, ce qui explique sa polysémie, correspondant à un processus interne à l'action publique (participation institutionnalisée, cf. Revel et al. 2007) ou au contraire à une dynamique exogène, sous une forme souvent contestataire. La participation recoupe la mise en place de mécanismes délibératifs, sans pour autant s'y réduire, et concerne tant des démarches top down que bottom up, des approches autogestionnaires que des dispositifs au service d'une libéralisation des services (JAGLIN 2006), et s'illustre par une diversité de dispositifs, du forum local au budget participatif (BACQUE, ReY \& SinTOMER 2005).

La participation est mise en avant dès les années 1960 en France, lorsque les savoirs experts acquièrent une place prépondérante dans la planification (ОвLEт 2005), posant la question de la place des citoyens dans le processus décisionnel. Elle est alors présentée comme un antidote à une action politique opaque, confisquée aux mains d'une oligarchie élue et/ou technique qui détient le monopole de la définition des objectifs et modalités de la transformation des territoires. Mis en place en réaction à l'urbanisme fonctionnel et à la seule planification, le projet urbain «suppose la participation active de tous les acteurs urbains, y compris les habitants» (Tomas 1998: 20). Analysant projets de ville et projets urbains, PINSON (2004) en relève la nature désormais «interactionniste et processuelle», impliquant une co- élaboration. La notion de régulation urbaine, adaptée des travaux de l'école régulationniste en économie (AglietTa 1997), désigne «les modes de production de la spatialité urbaine» (ABRAMO 1996), à travers règles et normes, dans leur production, mise en œuvre et infléchissement. La participation constituerait alors une dimension essentielle de la régulation contemporaine des territoires, engageant un rapport spécifique à l'élaboration des décisions. Ainsi, la participation émerge comme contrepoint de la complexification des savoirs, posant la question des capacités à participer à la vie publique et des savoirs engagés (CANTELLI et al. 2006). La participation indique-elle alors une autre articulation entre action publique et savoirs, laissant place à d'autres rapports réflexifs à la ville?

La démarche participative vient toutefois compléter et non contredire la démocratie représentative (RACINE 2008), dans la mesure où elle est généralement organisée par le pouvoir élu. Cette participation est-elle alors un instrument efficace de redistribution de la capacité décisionnaire en dehors des périodes électorales, introduisant des mécanismes délibératifs collectifs au cœur de la démocratie représentative, ou est-elle un leurre, relevant d'une «démocratie participative d'apparat» permettant le maintien d'une gestion urbaine oligarchique (Thomas 2003)? L'hypothèse d'une manipulation par la mise en place de processus participatifs est souvent posée, par exemple en Colombie (Lulle 2002) ou au Chili (Mosovich Pont-Lezica 2006). Ainsi la participation relève des opérations de légitimation de l'action publique, que cela en constitue un paravent ou une modalité essentielle.

À partir de l'étude de projets urbains à dimension patrimoniale à Gênes et Valparaiso, il s'agira de questionner la participation et ses modalités, en mettant en œuvre une démarche comparative, de façon à saisir dans des situations différentes les enjeux invariants. Les résultats présentés ici reposent sur des terrains de recherches de plusieurs mois menés à Gênes et à Valparaiso entre 2003 et 2008, impliquant un suivi des projets participatifs basé sur l'observation des projets, des entretiens avec les acteurs concernés, avant et après la réalisation des projets.

Gênes et Valparaiso sont marquées par le réinvestissement des espaces historiques centraux et portuaires depuis les années 1980, via un développement immobilier, touristique et culturel, stratégie choisie par de nombreuses autres villes industrialo-portuaires en 
crise (FAINSTEIN 1996; Gravari-Barbas 2007), et qui culmine dans les deux villes par une inscription au Patrimoine mondial de l'UNESCO, en 2003 à Valparaiso et en 2006 à Gênes. Les projets patrimoniaux sont intégrés aux projets de ville (Piano della Città de 1999 à Gênes, Cabildo à Valparaiso en 1992), permettant une réappropriation d'espaces délaissés et une diversification de l'économie urbaine, via le tourisme par exemple. Dans cette recherche, les projets urbains à dimension patrimoniale ont été privilégiés, le patrimoine constituant une construction sociale et culturelle (VeschAmbRe 2007) reposant sur des valeurs, impliquant un changement dans le statut des espaces et faisant l'objet de débats voire de conflits (MELE 2005). L'expression de ces valeurs patrimoniales lors de processus participatifs facilite la mise en avant d'options de développement urbain concurrentes. Cette étude se place alors dans une optique régulationniste, permettant d'identifier la façon dont la participation légitime ou contredit les options de développement urbain, accompagnant un régime urbain ou en favorisant la formation d'une nouvelle alliance urbaine, participant ainsi à la restructuration des «power structures» (DiGAETANo \& KLEMANSKI 1999). Les interventions urbaines dans le champ patrimonial constituent en outre un terrain d'application de nouveaux outils et méthodes de l'aménagement: démarche de projet, partenariats publics-privés, urban entrepreneurialism (Harvey 1989) et mise en avant de la nécessaire participation des acteurs et habitants. La participation est-elle exclusivement au service de la légitimation des stratégies publiques de développement ou permet-elle un infléchissement des projets menés?

En portant attention aux savoirs et aux compétences, la première partie est consacrée aux registres encadrés de la participation. Puis seront étudiés les liens entre participation et contestation, permettant de déceler les conditions d'une co-définition des choix du développement urbain et de montrer in fine la nécessaire articulation entre dimension axiologique et mise en avant de compétences expertes, justifiant la prétention à se situer en amont des projets proposés à la participation, quitte à entrer dans un processus participatif à partir d'une conflictualité initiale. Les participations suscitées par des collectifs d'habitants posent ainsi la question des enjeux mêmes du développement urbain.

\section{La conduite de la participation dans les projets de ville et urbains}

La participation relève désormais d'un des instruments utilisés dans le cadre des transformations urbaines. Encadrée et conduite par des professionnels, permet-elle la production d'un consensus sur les voies de l'aménagement?

\subsection{L'apprentissage de la participation}

Les programmes de formation à la participation indiquent que les acteurs publics posent en préalable à la participation l'acquisition de compétences, conçues comme élément de la citoyenneté. Dans ce cadre, la participation, centrée sur des opérations modestes, est sollicitée autant pour le résultat matériel - l'aménagement d'espaces urbains -, que pour la promotion d'une «citoyenneté active», qui suppose l'adhésion aux programmes publics et à leurs principes.

L'aménagement de la Piazza Ragazzi di Vico Indoratori dans le centre historique de Gênes constitue un exemple de participation incluant un apprentissage citoyen. Ce projet d'aménagement participatif d'un espace public est encadré par un service municipal traitant de la dimension sociale de la transformation urbaine (Terza Età Sicura, dirigé par la sociologue CARLA CoSTANZI). Le programme implique des habitants-volontaires, après un atelier de formation à la participation intitulé «citadinanza activa» (citoyenneté active). Cette expérience a concerné une dent creuse, issue des bombardements de la Seconde Guerre mondiale, d'une surface peu importante dans le centre historique, fréquentée alors par des Sud-Américains qui s'y réunissaient, selon divers habitants, pour des pratiques festives et alcoolisées. Le but est de réaménager cette place pour attirer de nouveaux usagers et éviter ceux considérés comme perturbateurs: fermeture de la place le soir, organisation d'animations pour enfants. Les volontaires autogèrent cet espace. Ici la participation a impliqué des habitants en accord avec la municipalité et ses objectifs de normalisation de l'espace et pas les usagers antérieurs. La sélection a priori des participants potentiels (Clayes-MeKDADE 2001) est une fermeture quant aux choix possibles.

De même, à Valparaiso, les diverses opérations de participation impliquent la formation des citadins, en suivant une méthodologie définie par les instances publiques nationales, via des manuels diffusés localement, comme le Secretaría de Medio Ambiente, TerritoRio y PARTICIPACIÓN CiUdAdANA - SEMAT (2003) par exemple: partition des acteurs selon leurs rôles, ateliers de discussion et de formation reprenant les discours de l'empowerment («empodamiento»), «image-cible» à partir de grilles SWOT («Strengths, Weaknesses, Opportunities and Threats»), consultants spécialisés en animation de participation.

Cette méthodologie se déploie à diverses échelles. Par exemple, une démarche de participation encadrée par atelier a été menée par le service du patrimoine de la commune en 2006 dans le quartier de Cerro Cordillera (une colline appartenant au périmètre défini par l'UNESCO), pour un projet limité de valorisation culturelle et touristique du quartier. Le projet, qui aboutit à la mise en place de panneaux rapidement 
décrochés, vise à renforcer l'adhésion des habitants à l'objectif de valorisation du patrimoine. À plus large échelle, les grands chantiers urbanistiques menés par la commission présidentielle Plan Valparaiso (devenu en 2006 Valpo Mío) incluent des processus participatifs, suite aux critiques locales d'un processus exogène à la ville. Les ateliers incluent la formation des citadins à la participation dans le programme Valparaiso Opina. Ici la participation cible les acteurs-relais (présidents des comités de quartier, représentants des diverses corporations) tandis que l'objet de la participation concerne des programmes déjà établis.

La participation vise dans ces différents cas autant la transformation d'un espace que la formation des citadins à une participation limitée à des opérations mineures, dont les objectifs confirment les stratégies globales. Dans le derniers cas, la participation est un palliatif aux critiques sur le manque de légitimité démocratique des opérations, sans questionner davantage les principes de l'aménagement. Ces processus participatifs présupposent ainsi une adhésion préalable aux orientations générales.

La participation est également utilisée en aval de la décision. Selon Bruno Gabrieldi, adjoint au maire de Gênes entre 1997 et 2007, «participer, c'est participer à l'action» (entretien 2006) et, par capillarité, la diffuser à l'ensemble du territoire. Dans cette optique, participer équivaut pour les propriétaires d'appartements qualifiés d'édifices historiques à participer aux programmes en profitant des incitations fiscales et en accompagnant les investissements publics. La participation se décline sous le registre de l'enrôlement des habitants, comme une adhésion active à l'action publique, et pas comme un mécanisme délibératif. Le consentement et la production d'un consensus sont suscités parfois sous la forme de l'enthousiasme. Il s'agit d'un passage à la limite de la notion de participation, qui apparaît ainsi «dépolitisée» (JAGLIN 2005).

Cet enrôlement constitue-t-il le motif de la mise en place d'une participation institutionnalisée? Les soupçons d'instrumentalisation de la participation tiennent là, qu'il y ait ou non délibération, à l'absence de discussion sur les fondements de la discussion: orientations stratégiques générales et valeurs les sous-tendant. La participation peut-elle se déployer dans un cadre plus global et concerner les principes mêmes du développement urbain?

\subsection{La mobilisation des acteurs de la ville et la construction d'un consensus}

Le projet de ville pour la définition des orientations stratégiques implique de nouveaux mécanismes décisionnels de «mobilisation sociale» (PINSON 2004), en partant du principe que la démarche de projet ne peut s'appuyer seulement sur des savoirs experts mais doit s'ouvrir aux ressources et visions des acteurs. Le terme «mobilisation» (OBLET 2005; Pinson 2004, 2009) signifie une forme d'action collective ayant pour but la production d'un consensus via une implication active des acteurs et citadins dans la définition du projet urbain (SALIGNON \& Younes 1998).

Cette mobilisation des acteurs est-elle conforme à l'acceptation du terme participation? Les participants sont là les acteurs clefs de la ville, représentants des institutions, entreprises et associations de poids au niveau local et moins les citoyens-citadins: ils n'incarnent ainsi pas la démocratie représentative de type électoral mais un compromis entre représentation et participation. Ce ne sont pas les citadins directement qui participent à la définition des orientations stratégiques mais les représentants des structures corporatives.

La réalisation du projet de ville de Gênes vise à impliquer de façon symbolique l'ensemble de la ville. En mai 1999 est organisée une Conférence stratégique avec différents acteurs politiques, économiques, associatifs, culturels et médiatiques aboutissant au plan stratégique, et non les citadins ordinaires, dans une démarche de mobilisation. En outre, la municipalité met en ouvre des conférences d'actualisation, notamment en 2005 , et des rencontres thématiques sur le tourisme en 2006. La mobilisation rassemble l'ensemble des acteurs sous le leadership de la municipalité, en figurant l'unité symbolique de la ville conçue comme acteur collectif transcendant les oppositions. En ce sens, le projet urbain et le processus participatif basé sur une mobilisation des acteurs clefs constituent un instrument du gouvernement urbain (LASCOUMES \& LE GaLES 2004), permettant la production d'un consensus politique sur les voies de développement choisies.

Trois figures de la participation ont ainsi pu être individualisées: participation localisée, enrôlement, mobilisation. Ces divers éléments tracent une vision en partie instrumentalisée de la participation, lorsqu'elle est décrétée en amont. Les habitants et acteurs ne sont pas perçus comme dépositaires de savoirs alternatifs puisque la participation est conçue comme un processus formatif. La participation est-elle alors possible sous une forme institutionnalisée sans être réduite à une démarche ponctuelle sans enjeux ou à une pratique réservée aux acteurs clefs en vue de l'obtention d'un consensus? Ce travail fait l'hypothèse qu'une participation efficace implique une démarche conflictuelle préalable à une institutionnalisation conduite au risque de l'altération des politiques publiques conduites.

\section{De la contestation à la participation}

La participation implique-t-elle une adhésion au paradigme porté par l'acteur exerçant le leadership? 
Les contestations urbaines sont-elles le creuset d'une participation correspondant à une démarche de type bottom up, préalable à une institutionnalisation? Les contestations urbaines à l'occasion de conflits patrimoniaux font apparaître des modalités de participation non réductibles à une instrumentalisation.

Le patrimoine est une construction sociale qui relève de valeurs multiples (RIEGL 1984) portant sur la définition du patrimoine et son usage. En effet, le patrimoine est lié à des processus d'appropriation qui définissent les propriétaires et les usagers légitimes (VESCHAMBRE 2008). Le patrimoine est ainsi souvent débordé par des enjeux plus larges concernant les usages et usagers légitimes des espaces, tandis que les conflits patrimoniaux sont porteurs de projets urbains concurrents et de valeurs urbaines alternatives (JACQUOT 2009). Dès lors, l'étude des processus contestataires autour des enjeux patrimoniaux peut-elle faire apparaître des processus participatifs émergents?

\subsection{La participation par l'institutionnalisation des contestations urbaines}

À Gênes, avant le réinvestissement du centre historique, se développent à la fin des années 1980 et au début des années 1990 des comités d'habitants qui, pour attirer l'attention des autorités publiques sur les problèmes quotidiens du centre historique, rédigent des lettres ouvertes et des pétitions. Au départ, le patrimoine ne constitue pas un motif d'action: ce qui est en jeu dans ces premières contestations est la priorité accordée par les pouvoirs publics aux monuments, au détriment des conditions de vie des habitants. Ces premières contestations bousculent ainsi l'ordre des priorités identifiées.

Les comités formés en 1989 fonctionnent par quartiers avec plus de 300 personnes, auxquels s'ajoutent les paroisses et l'Union des commerçants du centre historique. Un groupe d'habitants prend en charge la coordination et publie en 1990 un Livre Blanc. La contestation prend une dimension globale, avec la dénonciation de l'état d'hygiène du centre historique, la délinquance, le délabrement du bâti, les ruines datant de la Seconde Guerre mondiale, la prostitution, etc. Le mouvement souhaite considérer le centre historique comme un ensemble unitaire («insieme unitario») (LiBro Bianco 1990), porteur de valeurs spécifiques. Ainsi ce mouvement constitue un territoire par son organisation et l'échelle de ses revendications.

Le processus participatif se resserre sur une vingtaine d'habitants qui cherchent à formaliser une démarche participative en créant l'Associazione per la Rinascita del Centro Antico (ARCA), voulant dépasser la contestation pour proposer des interventions. Un nouvel ouvrage intitulé Libro Verde sul centro storico (ARCA
1992) présente une posture réflexive, avec des passages théoriques sur la participation, du fait de la présence d'urbanistes et d'architectes, au courant des expériences urbaines menées dans diverses villes européennes, notamment Lyon ou Nantes. Dans cet esprit, la participation désigne une institutionnalisation de la démarche. Le fossé entre acteurs publics et comités d'habitants est résorbé par une conception de la participation soluble dans les modalités d'interventions des pouvoirs publics.

ARCA accentue cette démarche participative en proposant un programme d'intervention sur le quartier de Piazza delle Vigne, autour de son église et de son clồtre du X $\mathrm{X}^{\mathrm{e}}$ siècle. Ce quartier, au milieu des années 1990 , est emblématique des problèmes du centre historique: délinquance, prostitution, logements délabrés. ARCA fonctionne comme une équipe de coordination, organisant des réunions avec les institutions publiques (mairie, surintendance, région), les habitants et les propriétaires, pour les convaincre de se joindre à la réhabilitation. L'une des animatrices du projet, l'architecte-urbaniste Marolina Besio (1999), développe les principes de ce programme en les opposant à ceux de l'intervention publique habituelle. Elle met en avant la nécessité de partir de projets locaux, portés par les acteurs publics associés aux habitants et acteurs privés, à partir d'actions multidimensionnelles et de financements multiples. La démarche participative est en outre légitimée par les actions multiples («autorecupero») des habitants et propriétaires qui, hors de tout cadre institutionnel, agissent dans leur environnement (BEsIo 1999), rejoignant l'idée d'une participation par l'action.

Accueilli favorablement, le projet d'intervention est financé par un programme régional, le Programma Organico di Intervento (POI), permettant la restauration de l'église et de son cloître, la réhabilitation des espaces publics et la prise en charge d'une partie des travaux privés. Ainsi, ce mouvement d'abord contestataire, né de la colère de centaines d'habitants, est l'objet d'une institutionnalisation qui en marque le couronnement (par le succès d'une opération qui en reprend les principes) autant que la fin, aucun autre processus participatif endogène n'ayant été mené par la suite. La participation quitte donc le registre de la contestation pour construire, sur la base de compétences techniques de certains de ses membres, un modèle d'intervention, perdant son caractère populaire. Dynamique née dans la contestation, son institutionnalisation est facilitée par une communauté d'objectifs entre action publique et action citadine.

\subsection{La participation, source de nouveaux principes de l'action publique locale}

De même, à Valparaiso, la participation est née au creuset de contestations sur le devenir urbain et patri- 
monial de la ville. Dans les années 1990 se constitue un comité intitulé Ciudadanos por Valparaíso, opposé aux transformations du bâti dans les espaces historiques. Ce comité est structuré autour d'architectes et d'urbanistes, qui proposent une vision alternative du développement urbain. Souhaitant acquérir un poids politique, Ciudadanos intervient auprès des habitants. Une première campagne a pour thème la qualité de vie et la préservation du patrimoine via le droit à la vue de tous, vers la mer ou le reste de la ville, ce qui suppose un contrôle des hauteurs du bâti. Une seconde campagne se penche sur la conservation des commerces populaires dans le cadre de la transformation urbaine. La participation concerne ainsi des enjeux localisés, à partir d'un groupe actif qui tente d'accroître sa légitimité par l'assentiment des habitants.

Les débats sur le devenir du front de mer de la ville, enjeu majeur dans les années 2000, sont l'occasion pour Ciudadanos de peser de façon accrue en proposant une vision alternative. La participation contribue là à une réorientation radicale de la stratégie de développement, passant de projets immobiliers de grande envergure en front de mer à une régulation accrue par la patrimonialisation. L'étude des alliances et coalitions urbaines (DiGaetano \& KLEMANSKi 1999) permet de comprendre l'impact des processus participatifs, en les reliant à la question du pouvoir. Une coalition d'acteurs est mue par des principes et un horizon d'action (l'agenda).

À Valparaiso se forment deux coalitions. Une première coalition, constituée par des acteurs nationaux (l'entreprise portuaire, le Gouvernement, la Région) et internationaux (les investisseurs sollicités), repose sur la croissance urbaine via les investissements immobiliers, caractéristique des coalitions pro-croissance (Logan \& Molotch 1987), avec pour objectif le projet immobilier Barón, en front de mer, sur des friches portuaires et ferroviaires. Toutefois, se forme une coalition concurrente de régulation de la croissance dans toute la ville, au nom d'une conception unitaire de Valparaiso et d'un refus d'un développement immobilier exogène en hauteur qui menace les paysages urbains traditionnels. L'association Ciudadanos por Valparaiso joue un rôle essentiel dans cette coalition, d'abord en s'opposant aux projets de construction de tours, pour des motifs paysagers, puis en théorisant les raisons de ce refus, au nom du développement endogène et du droit de tous aux aménités urbaines. Peu à peu, la municipalité valide cet argumentaire, étendant la protection paysagère à l'ensemble de la baie en créant des zones de conservation patrimoniale en 2004, qui ont pour effet une protection paysagère de la baie de Valparaiso, définissant des hauteurs maximales de constructions à partir de points de vue appelés miradors. Le projet Barón, en dépit de la coalition qui le soutient, est ainsi fortement infléchi par la définition de nouvelles normes, puis par la controverse sur la conservation d'un entrepôt jugé de valeur patrimoniale. Là, l'institutionnalisation ne concerne pas l'action de l'association mais ses principes et valeurs, qui ont irradié l'action urbanistique locale (carte 1).

Dans ces deux cas, la participation naît d'un processus contestataire non réductible à une instrumentalisation, car l'intervention publique a été modifiée par un processus au départ exogène. Toutefois, à Gênes, la participation a été internalisée au sein de l'action publique, du fait d'une convergence d'objectifs, tandis qu'à Valparaiso elle a été un vecteur de principes alternatifs, qui ont infléchi les modalités de l'intervention urbaine.

\section{Des savoirs experts et des valeurs, quelle légitimation?}

Les dispositifs participatifs ont été mis en place pour répondre aux critiques formulées à la démocratie représentative. Alors qu'est souvent dénoncé le mirage de la participation (Thомas 2003), cette dernière se déploie efficacement lorsqu'elle repose sur des expertises multiples (Blondiaux \& Michel 2006) et relève d'une démarche contestataire préalable, motivée par des valeurs concurrentes, comme l'illustre le cas de Valparaiso.

Les processus participatifs ont eu un impact plus important lorsqu'ils s'appuient sur une contestation initiale, creuset de nouvelles conceptions du développement urbain. Or, dans les deux cas étudiés, ces processus s'appuient également sur des savoirs experts qui ont permis la médiation avec l'action publique et qui fondent sa prétention à la contestation. La remise en cause du projet Barón a été menée par Ciudadanos à partir d'une application stricte des documents d'urbanisme et de la législation patrimoniale et d'une contestation des procédures d'aménagement, en posant des recours (en lien avec le Comité de défense de Valparaiso, un regroupement d'associations locales et nationales). De même, à Gênes, ARCA s'appuie sur des expertises en urbanisme et en procédures participatives.

Ces actions mobilisent également des valeurs. L'opposition au projet Barón à Valparaiso repose sur la promotion d'un urbanisme qui s'inscrit dans les formes héritées et d'une ville égalitaire et accessible à tous (les tours étant perçues comme une confiscation des paysages par une minorité). Deux dimensions semblent ainsi nécessaires à une participation infléchissant les politiques publiques: une dimension experte et une dimension axiologique. 


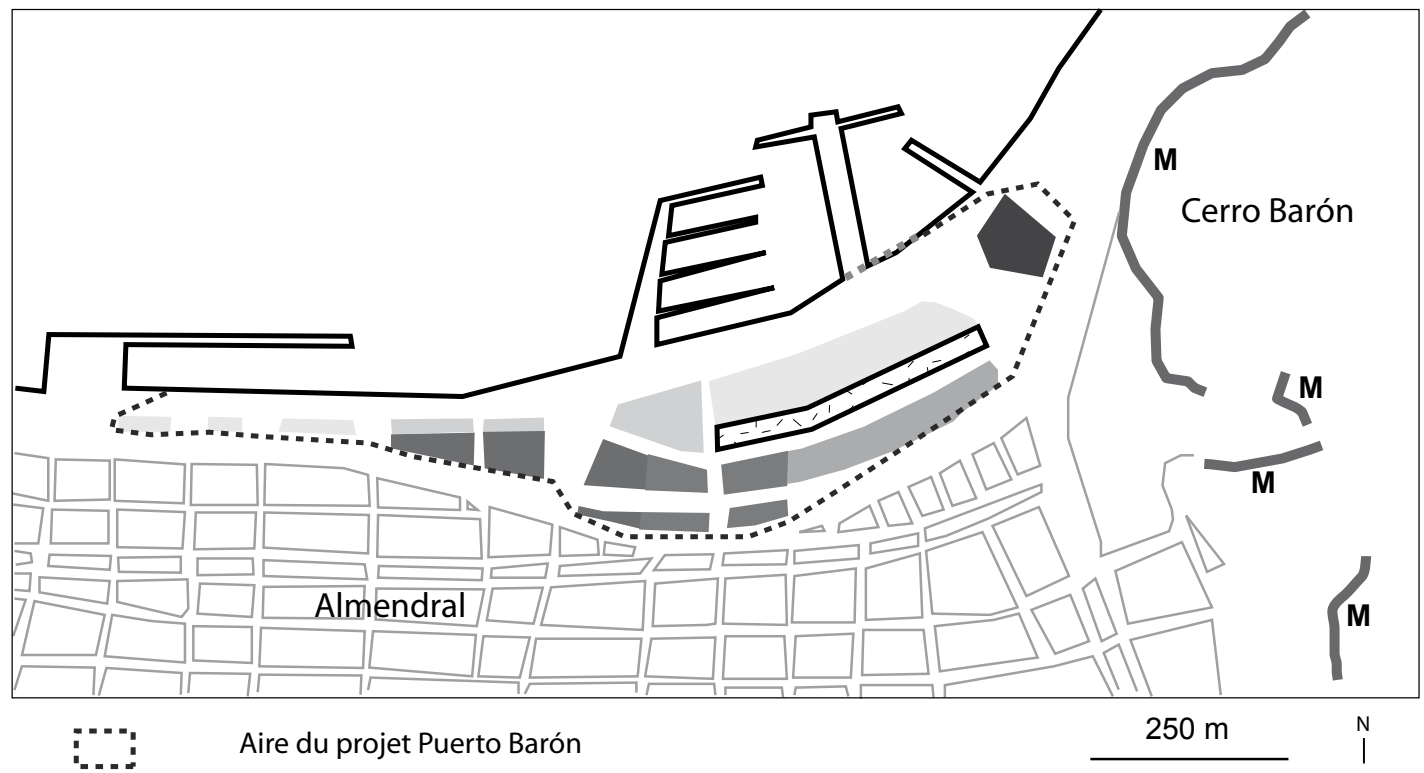

\section{Les obstacles au développement} autonome du projet Puerto Barón

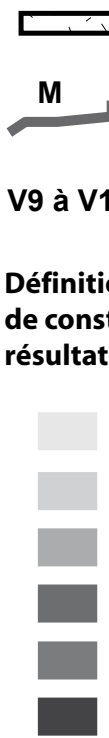

Entrepôt Simon Bolivar (édifice patrimonial)

Mirador (promenades avec points de vue)

Zones de détermination des hauteurs à partir du mirador Barón

Définition des hauteurs maximales de construction (zone V / hauteur max.), résultat de la régulation patrimoniale

$V 9 / 9,0 \mathrm{~m}$
$\mathrm{~V} 10 / 15,0 \mathrm{~m}$
$\mathrm{~V} 11 / 21,0 \mathrm{~m}$
$\mathrm{~V} 12 / 24,0 \mathrm{~m}$
$\mathrm{~V} 13 / 34,0 \mathrm{~m}$
$\mathrm{~V} 14 / 37,5 \mathrm{~m}$

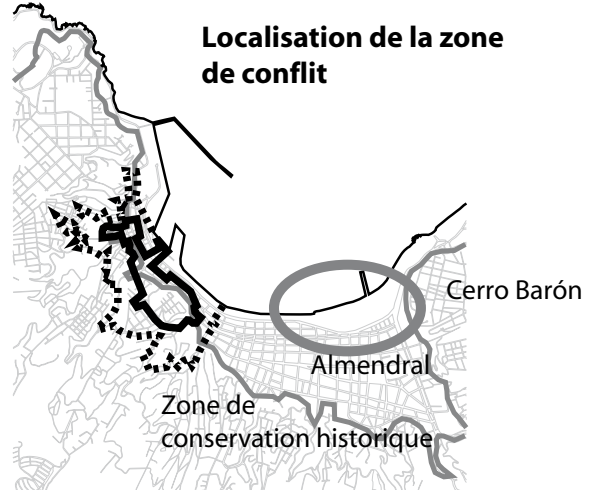

Limite de la plaine littorale

- Secteur Unesco

.... Zone tampon

Carte 1: Participation, conflits et régulation de la croissance: le projet Barón à Valparaiso, Chili Partizipation, Konflikte und Regelung des Wachstums: das Projekt Barón in Valparaiso, Chile Participation, conflict and regulation of growth: Barón Project in Valparaiso, Chile Source: Ordenanza Local Plan Regulador de Valparaíso, 2005; fonds de carte et cartes réalisées par S. JaCQUOT 
Cette mise en avant d'une confrontation des valeurs permet de dépasser l'opposition entre intérêt local et intérêt général dans les processus participatifs, faisant émerger un citoyen-riverain (FourNIAU 2007), capable à partir d'un enjeu local d'aboutir à l'énoncé de valeurs qui débordent ce cadre spatial, comme l'illustrent les controverses patrimoniales, occasion dans les deux villes comparées de reconstruire des valeurs induisant un devenir urbain différent. Cela suppose d'argumenter sur la légitimité de ce citoyen-riverain à se saisir d'une question plus vaste et, à partir de l'enjeu localisé qui motive son action, à énoncer des valeurs de portée générale. La participation engage ainsi, outre la discussion sur l'intervention urbaine, une discussion sur les compétences et légitimités et opère une redistribution de la capacité à formuler des enjeux entre élus et citoyens.

\section{Bibliographie}

Aвramo, P. (1996): La régulation et le régime urbains. La structure urbaine, sa reproduction et le capital. - In: Futur Antérieur 30, http://multitudes.samizdat.net/Laregulation-et-le-regime-urbains 15.12.2009.

Aglietta, M. (1997): Régulation et crises du capitalisme. - Paris: Odile Jacob.

Associazione per la Rinascita del Centro Antico - ARCA (1992): Libro verde sul centro storico. Genova: SAGEP.

Bacque, M.-H., Rey, H. \& Y. Sintomer (dirs) (2005): Gestion de proximité et démocratie participative, une perspective comparative. - Paris: La Découverte.

Besio, M. (1999): Il libro delle Vigne, dalla parte degli abitanti. Un progeto di riqualificazione urbana a Genova. - Torino: Unmerto Allemandi \& Co.

Blatrix, C. (2002): Devoir débattre. Les effets de l'institutionnalisation de la participation sur les formes de l'action collective. - In: Politix 15, 57: 79-102.

Blondiaux, L. \& L. Michel (2006): L'expertise en débat, jeux d'acteurs et conflits de savoirs autour d'un débat public local dans le Lot. - In: Cantelli, F., JacoB, S., Genard, J.-L. \& C. DE VISSChER (dirs): Les constructions de l'action publique. - Paris: L'Harmattan: 181-201.

Cantelli, F., Jacob, S., Genard, J.-L. \& C. De Visscher (dirs) (2006): Les constructions de l'action publique. - Paris: L'Harmattan.

Clayes-Mekdade, C. (2001): Qu'est-ce qu'une «population concernée»? L'exemple camarguais. - In: Géocarrefour 76, 3: 217-223.

DiGaetano, A. \& J.S. KLEMANSKI (1999): Power and city governance: Comparative perspectives on urban development. - Minneapolis: University of Minnesota Press. FAINSTEIN, S. (1996): The changing world economy and urban restructuring. - In: FAINSTEIN, S. \& S. CAMPBELL (dirs): Readings in urban theory. - Oxford: Blackwell. FourniaU, J.-M. (2007): Citoyen en tant que riverain: une subjectivation politique dans le processus de mise en discussion publique des projets d'aménagement.
- In: Revel, M., Blatrix, C., Blondiaux, L., Fourniau, J.-M., Heriard Dubreuil, B. \& R. Lefebvre (éds): Le débat public, une expérience française de démocratie participative. - Paris: La Découverte: 67-77.

Gravari-Barbas, M. (2007): De la fête dans la ville à la ville festive. Les faits et les espaces festifs, objet géographique émergent. - In: DA CUNHA, A. \& L. MATTHEY (éds): La ville et l'urbain: des savoirs émergents? Perspectives critiques et méthodologiques. - Lausanne: Presses Polytechniques et Universitaires Romandes.

Harvey, D. (1989): From managerialism to entrepreneurialism: the transformation in urban governance in late capitalism. - In: Geografiska Annaler: Series B, Human Geography 71, 1:3-17.

JACQUOT, S. (2009): Les figures de la ville, éléments de compréhension des débats sur la ville. - In: Vallat, C., Dufaux, F. \& S. Lehman-Frisch (éds): Pérennité urbaine, la ville par-delà ses métamorphoses. Volume 3. - Paris: L'Harmattan: 165-177.

JAGLIN, S. (2005): La participation au service du néolibéralisme? Les usagers dans les services d'eau en Afrique subsaharienne. - In: BACQue, M.-H., ReY, H. \& Y. Sintomer (dirs): Gestion de proximité et démocratie participative, une perspective comparative. - Paris: La Découverte: 271-293.

Lascoumes, P. \& P. Le Gales (2004): Gouverner par les instruments. - Paris: Presses de Sciences Po.

LE Gales, P. (2003): Le retour des villes européennes. - Paris: Presses de Sciences Po.

Legros, O. (dir.) (2008): Participations citadines et action publique: Dakar, Rabat, Cotonou, Tunis, Jérusalem, Sanaa. - Gap: Editions Yves Michel.

LiBRo BIANCO (1990): Genova, libro bianco sul centro storico. - Genova: SAGEP.

Logan, J. \& H. Molotch (1987): Urban fortunes. The political economy of place. - Berkeley: University of California Press.

Lulle, T. (2002): A qui sert la gestion participative locale? Eléments de réflexion à partir d'une expérience à Bogota. - In: Autrepart 21:151-168.

Mele, P. (2005): Conflits patrimoniaux et régulations urbaines - In: ESO Travaux et documents. Patrimoine et développement durable, les villes face au défi de la gouvernance territoriale 23: 51-57

Mosovich Pont-Lezica, D. (2006): La lutte pour le logement en temps de la globalisation. Le cas du Proyecto Ribera Norte, Concepcion, Chili. - In: Géocarrefour 81, 2: 121-125.

OвLEт, T. (2005): Gouverner la ville. - Paris: Presses Universitaires de France.

Pinson, G. (2004): Le projet urbain comme instrument d'action publique. - In: Lascoumes, P. \& P. Le Gales (dirs): Gouverner par les instruments. - Paris: Presses de Sciences Po: 199-233.

Pinson, G. (2009): Gouverner la ville par un projet. Urbanisme et gouvernance des villes européennes. - Paris: Presses de Sciences Po. 
Racine, J.-B. (2008): Projet urbain et démarche participative: contribution géographique à la possibilité de choisir sa ville aujourd'hui, quelques leçons de l'expérience lausannoise. - In: Bulletin de la société géographique de Liège 50: 5-15.

Revel, M., Blatrix, C., Blondiaux, L., Fourniau, J.M., Heriard Dubreuil, B. \& R. Lefebvre (2007): Le débat public, une expérience française de démocratie participative. - Paris: La Découverte.

RIEGL, A. (1984): Le culte moderne des monuments. - Paris: Seuil.

SAlignon, B. \& C. Younes (1998): La médiation comme ouverture au projet urbain. - In: TousSAINT, J.-Y. \& M. ZimMERMANN (dirs): Projet urbain, ménager les gens, aménager la ville. - Sprimont: Mardaga: 175-183.

Secretaría de Medio Ambiente, Territorio y Participación Ciudadana - SEMAT (MOP) (2003): Manual de Participación ciudadania. - Gouvernement du Chili: Editions LOM.

Sintomer, Y. \& L. Blondiaux (2002): L'impératif délibératif. - In: Politix 15, 57: 17-35.

Thomas, O. (2003): Gouvernement des villes et démocratie participative: quelles antinomies? - In: Pouvoirs 1, $104: 143-158$.

Tomas, F. (1998): Vers une nouvelle culture de l'aménagement des villes. - In: ToussaINT, J.-Y. \& M. ZIMMERMANN (dirs): Projet urbain, ménager les gens, aménager la ville. - Sprimont: Mardaga: 13-34.

Veschambre, V. (2007): Le patrimoine: un objet révélateur des évolutions de la géographie et de sa place dans les sciences sociales. - In: Annales de géographie 656:361-381. Veschambre, V. (2008): Traces et mémoires urbaines, enjeux sociaux de la patrimonialisation et de la destruction. - Rennes: Presses Universitaires de Rennes.

\section{Résumé: La participation au service de la régulation urbaine dans le cadre des projets urbains et patrimo- niaux à Gênes, Italie et à Valparaiso, Chili}

Cet article vise à présenter différents registres de la participation à Gênes et à Valparaiso, à partir de projets urbains à dimension patrimoniale. La participation est étudiée comme une modalité d'action collective intégrée à l'action publique et pose la question des processus de légitimation des projets et des capacités à formuler des projets, dans la mesure où la participation vise à permettre aux citoyens à peser sur l'aménagement urbain. Deux catégories de démarches participatives sont comparées: celles conduites par les pouvoirs publics, impliquant une définition a priori des principes de l'aménagement et celles résultant de processus contestataires. Deux dimensions semblent ainsi nécessaires à une participation infléchissant les politiques publiques: une pluralité d'expertises et une dimension axiologique.

Mots-clés: participation, projets urbains, légitimation, Gênes, Valparaiso
Zusammenfassung: Partizipation im Dienste der urbanen Regulierung im Rahmen von Städteplanung und Erhaltung städtischen Erbes in Genua, Italien und Valparaiso, Chile

Dieser Artikel stellt anhand von Städteplanungen zur Erhaltung städtischen Erbes verschiedene Modalitäten von Partizipation in Genua und Valparaiso dar. Partizipation wird hier als Form gemeinschaftlichen Handelns verstanden, das in den amtlichen Handlungsspielräumen integriert ist. Dies stellt die Legitimation von Projekten infrage sowie die Fähigkeit, diese zu formulieren. Partizipation bei Projekten sollte es den Bürgern ermöglichen, sich in die Stadtentwicklungsplanung einzubringen. Es werden zwei Arten von Partizipation verglichen: jene, die von den Behörden angeführt wird und von vornherein die Prinzipien der Einrichtungen festlegt, und jene, die sich aus Oppositionsprozessen ergibt. Zwei Aspekte sind notwendig für eine Partizipation, welche die öffentliche Politik beeinflussen kann: eine Vielzahl von Gutachten und eine axiologische Dimension.

Schlüsselwörter: Partizipation, Städteplanung, Legitimation, Genua, Valparaiso

\section{Summary: Participation as part of urban regulation in urban planning and heritage in Genoa, Italy and Valparaiso, Chile}

This article presents different approaches to participation using case studies of urban development and heritage projects in Genoa and Valparaiso. Participation is seen here as a form of collective action integrated into public action. Integration of participation affects the process of legitimisation of projects and ability to formulate projects as its purpose is to give citizens the opportunity to influence the urban planning process. Two types of participation are compared here: those led by public authorities, which imply a definition a priori of urban planning principles, and a participation process growing out of a movement of protest. It is argued that two aspects are necessary for participation to modify public policy: a plurality of expertises and an axiological dimension.

Keywords: participation, urban planning, legitimisation, Genoa, Valparaiso

Dr. Sébastien Jacquot, Institut de Recherches et d'Etudes Supérieures du Tourisme (IREST), Université de Paris 1 Panthéon Sorbonne, Centre Albert Châtelet, 6, rue Jean Calvin, F-75005 Paris, France.

e-mail: sebastien.jacquot@univ-paris1.fr

\section{Manuskripteingang/received/manuscrit entré le 6.7.2009}

Annahme zum Druck/accepted for publication/accepté pour l'impression: 18.12.2009 